\title{
SATB1-dependent mitochondrial ROS production controls TCR signaling in CD4 T cells
}

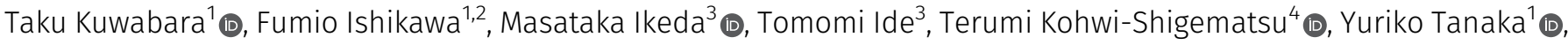 \\ Motonari Kondo 10
}

\begin{abstract}
Special AT-rich sequence binding protein-1 (SATB1) is localized to the nucleus and remodels chromatin structure in T cells. SATB1deficient CD4 T cells cannot respond to TCR stimulation; however, the cause of this unresponsiveness is to be clarified. Here, we demonstrate that SATB1 is indispensable to proper mitochondrial functioning and necessary for the activation of signal cascades via the TCR in CD4 T cells. Naïve SATB1-deficient CD4 T cells contain fewer mitochondria than WT T cells, as the former do not express mitochondrial transcription factor A (TFAM). Impaired mitochondrial function in SATB1-deficient T cells subverts mitochondrial ROS production and SHP-1 inactivation by constitutive oxidization. Ectopic TFAM expression increases mitochondrial mass and mitochondrial ROS production and rescues defects in the antigenspecific response in the SATB1-deficient T cells. Thus, SATB1 is vital for maintaining mitochondrial mass and function by regulating TFAM expression, which is necessary for TCR signaling.
\end{abstract}

DOI 10.26508/Isa.202101093 | Received 14 April 2021 | Revised 9 September 2021 | Accepted 10 September 2021 | Published online 28 September 2021

\section{Introduction}

T-cell activation is triggered by signals via the TCR upon recognition of the complex composed with antigen peptides and self-MHC (Nel, 2002; Nel \& Slaughter, 2002; Smith-Garvin et al, 2009). Lymphocyte-specific protein tyrosine kinase (LCK), an Src family tyrosine kinase, initiates downstream TCR signaling by phosphorylating the immunoreceptor tyrosine-based activation motif(ITAM) within the TCR-associated CD3ろchains (Molina et al, 1992; Straus \& Weiss, 1992). Phosphorylated ITAM generate docking sites for $70-k D$ $\zeta$-chain-associated protein kinase (ZAP70). Lck also phosphorylates ZAP70, which propagates signaling events such as intracellular calcium influx and the MAPK kinase known as Ras-MAPK or extracellular signal-regulated kinase (ERK) (van Leeuwen \& Samelson, 1999). Both of these events are necessary for T-cell activation (Smith-Garvin et al, 2009; Courtney et al, 2018; Gaud et al, 2018). Thus, regulation of Lck activity is critical for T-cell function.
A major negative regulator of Lck, which sets the TCR signaling threshold, is the tyrosine phosphatase SHP-1 (Kosugi et al, 2001). Aberrant Lck activation is observed in SHP-1-deficient mice leading to T-cell hyperactivation, increased IL-2 production, and autoimmunity (Carter et al, 1999; Lorenz, 2009). Furthermore, the TCR signal cascade cannot be activated in $T$ cells in the presence of the constitutive active form of SHP-1 (Štefanová et al, 2003; Capasso et al, 2010). Therefore, regulation of SHP-1 activity is crucial for T-cell activation. However, the regulatory mechanisms of SHP-1 activity in resting $T$ cells are not well-understood.

Mitochondria are the powerhouses of cells as they produce cellular energy sources such as adenosine $5^{\prime}$-triphosphate (ATP) (Mills et al, 2017). Mitochondria play key roles in the tricarboxylic acid (TCA) cycle and cellular respiration and participate in fatty acid synthesis, $\mathrm{Ca}^{2+}$ homeostasis, and heme and $\mathrm{Fe}-\mathrm{S}$ protein biogenesis (Tait \& Green, 2012). For mitochondrial biogenesis, Tfam, a nuclear gene encoding mitochondrial transcription factor A (TFAM), is essential for mitochondrial DNA transcription and replication (Fisher et al, 1989; Larsson et al, 1998; Nisoli et al, 2005). In various cell types, the multifunctional mitochondria are integrated into the cell signaling circuitry (Gunter et al, 2004; Chandel, 2010; Finkel, 2011; Arciuch et al, 2012; Tait \& Green, 2012). In T cells, mitochondria move near the TCR complex, which appear to be required for T-cell activation (Quintana et al, 2007; Baixauli et al, 2011). However, the precise functional roles of mitochondria in TCR signals remain unclear.

The chromatin structure modulates the accessibility of various transcription factors to the regulatory regions of target genes and regulates gene expression. Such chromatin topology is regulated by chromosomal organizers (Schneider \& Grosschedl, 2007; Phillips \& Corces, 2009). Matrix attachment DNA regions (MARs) or scaffoldassociated regions in DNA sequences may mediate chromatin loop formation, which is essential for genomic DNA compaction and chromatin organization into genomic functional units (Cockerill et al, 1987; Zlatanova \& van Holde, 1992). MARs often colocalize with enhancer regions and regulate both positive and negative gene expression (Banan et al, 1997; Zhang et al, 1998; Zhong et al, 1999). Special AT-rich sequence binding protein-1 (SATB1) is an

\footnotetext{
${ }^{1}$ Department of Molecular Immunology, Toho University School of Medicine, Tokyo, Japan ${ }^{2}$ Faculty of Health Sciences, Tsukuba International University, Tsuchiura, Japan ${ }^{3}$ Department of Cardiovascular Medicine, Graduate School of Medical Sciences, Kyushu University, Fukuoka, Japan ${ }^{4}$ Department of Orofacial Science, University of California San Francisco School of Dentistry, San Francisco, CA, USA
}

Correspondence: kuwabara@med.toho-u.ac.jp 
MAR-binding protein (Dickinson et al, 1992) and genome organizer. SATB1 anchors AT-rich DNA sequences to the nuclear matrix and forms genomic DNA loops (Cai et al, 2003; Kumar et al, 2007). SATB1 can also recruit chromatin-remodeling complexes and regulate gene expression (Kumar et al, 2007). SATB1 is necessary for the expression of several T helper genes and modulates effector T-cell function by holding the promoter regions of cytokine genes in close proximity (Cai et al, 2006). In addition, mice without SATB1 in their T cells failed to respond to antigens, albeit SATB1-deficient mice are prone to developing autoimmune disorders (Tanaka et al, 2017; Akiba et al, 2018). Accordingly, SATB1 is essential for T-cell activation; however, its contribution to T-cell function remains unknown.

In this study, we demonstrate that SATB1 regulates mitochondrial reactive oxygen species (mtROS) production and mitochondrial maintenance through the expression of TFAM protein. We also found that SATB1-dependent mtROS is necessary for modulation of SHP-1 activity and threshold setting for T-cell activation upon TCR stimulation. Therefore, SATB1 plays a vital role in TCR function by maintaining mitochondrial homeostasis. The insights obtained from this study will help in the development of treatment strategies for T-cell-mediated immune diseases.

\section{Results}

\section{SATB1 is indispensable in T-cell activation}

To determine the relevance of SATB1 protein in T-cell activation, we purified naïve CD4 T cells from wild type (WT) and SATB1cKO mice,
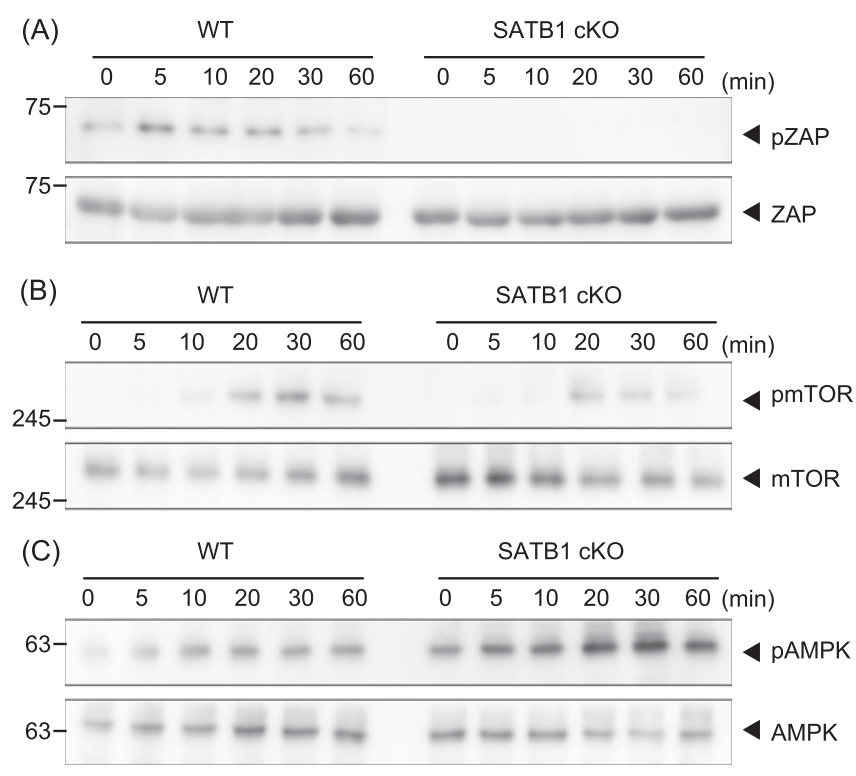

\section{Figure 1. SATB1 is required for T-cell activation.}

Signaling molecule phosphorylation was examined in naïve CD4 T cells from WT and SATB1CKO mice. (A, B, C) T cells were stimulated with anti-CD3 and anti-CD28 antibodies for the indicated time periods, lysed, analyzed by SDS-PAGE, and immunoblotted with anti-ZAP70, anti-phospho-ZAP70 (A), anti-mammalian target of rapamycin, anti-phospho-mammalian target of rapamycin (B), antiAMPK, and anti-phospho-AMPK (C). Data for one experiment are representative of three independent experiments. stimulated the cells with anti-CD3 and anti-CD28 antibodies, and examined the activation status via the bromodeoxyuridine (BrdU) uptake and DNA content. We found that $4.5 \%$ and $45 \%$ of T cells from WT mice were BrdU-positive at 24 and $48 \mathrm{~h}$ after TCR stimulation, respectively. In contrast, $T$ cells from SATB1cKO mice contained far fewer BrdU-positive cells (Fig S1A-C). Hence, SATB1 is required for T-cell proliferation by TCR-mediated stimulation in accordance with our previous reports (Tanaka et al, 2017; Akiba et al, 2018).

Next, we investigated whether SATB1-deficient T cells adequately phosphorylate the signaling molecules in the TCR cascade. In WT T cells, we detected ZAP70 phosphorylation in TCR-mediated signaling within 5 min of cross-linking with anti-CD3 and anti-CD28 antibodies (Fig 1A). However, T cells from SATB1cKO mice showed substantially lower ZAP70 phosphorylation than the WT control. These results demonstrate that SATB1 is necessary for triggering TCR signal cascades in CD4 T cells.

\section{Mitochondrial function is impaired in SATB1cKO T cells}

The mammalian target of rapamycin (mTOR) pathway plays an important role in T-cell activation (Maclver et al, 2013; Desdin-Mico et al, 2018). In accordance with the results shown above, mTOR phosphorylation was markedly reduced in T cells from SATB1cKO mice than those from WT mice after TCR stimulation (Fig 1B). In addition, phosphorylation of p70S6 kinase, a downstream molecule of mTOR was much lower in SATB1CKO CD4 T cells than in WT CD4 T cells (Fig S2). Because tonic signals via TCR are necessary for T-cell survival, we hypothesized that SATB1CKO CD4 T cells were under energetic stress, leading to activation of the serine/threonine AMPactivated protein kinase (AMPK). AMPK can be phosphorylated in T cells upon TCR stimulation. In fact, we observed increased AMPK phosphorylation levels in both WT and SATB1CKO T cells (Fig 1C). More importantly, AMPK was phosphorylated in the resting state of SATB1CKO cells but not WT T cells (Fig 1C), suggesting that SATB1cKO $T$ cells lack sufficient cellular energy, in which AMPK may be constitutively activated.

Cellular processes such as transcription and translation are enhanced in T cells after TCR stimulation. T-cell activation requires sufficient cellular energy such as ATP, suggesting that loss of mitochondria and short supply of ATP occur in SATB1-deficient T cells. Next, we examined the cellular ATP levels in CD4 T cells from WT and SATB1CKO mice. Primary CD4 T cells from SATB1CKO mice contained $60 \%$ less ATP than those from WT mice. The former also showed increased ADP/ATP ratios (Figs 2A and S3). Thus, SATB1 loss may cause severe energetic stress and mitochondrial dysfunction. Therefore, we investigated whether mitochondrial function is weaker in SATB1CKO than in WT T cells. The activity level of an NADH dehydrogenase in mitochondrial complex I was markedly lower in SATB1-deficient T cells than in WT T cells (Fig 2B). We prepared naïve CD4 T cells from WT and SATB1CKO mice, incubated them with the ROS-sensitive dye DCFDA, and measured intracellular ROS levels. As shown in Fig 2C, WT T cells had a distinct bimodal fluorescence distribution in the absence of TCR stimulation. In contrast, SATB1CKO CD4 T cells exhibited low unimodal fluorescence peaks (Fig 2C). TCR stimulation for $30 \mathrm{~min}$ increased the relative DCFDA fluorescence-positive WT T-cell population (Fig 2C and D). However, 
(A)

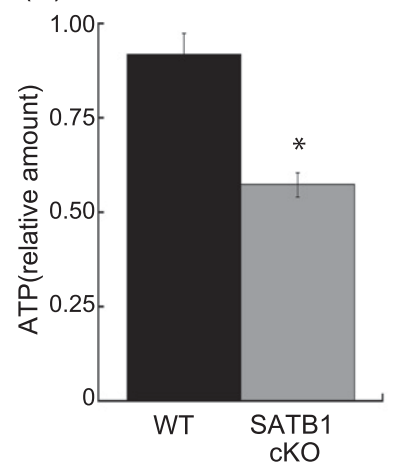

(C) WT

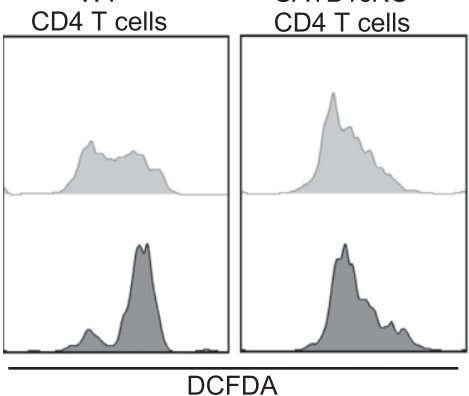

(D)

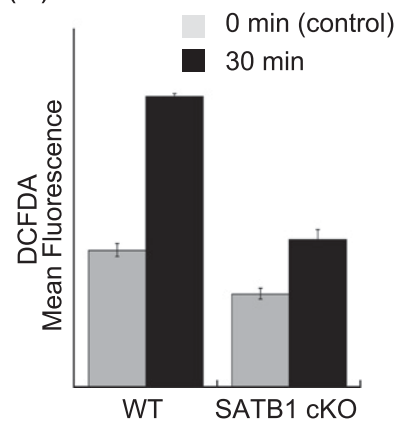

(B)
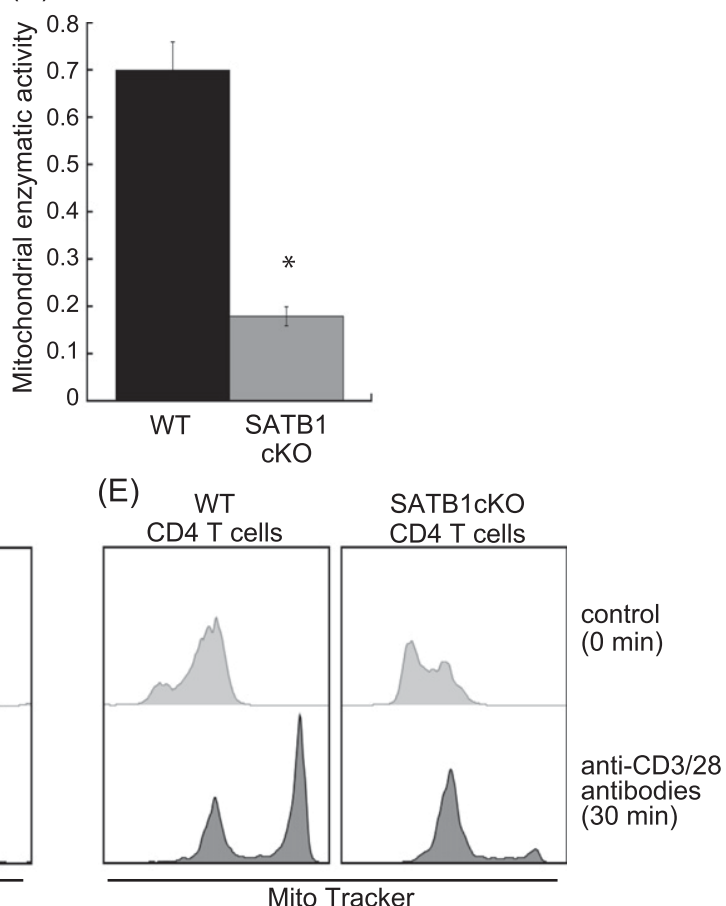

(F)

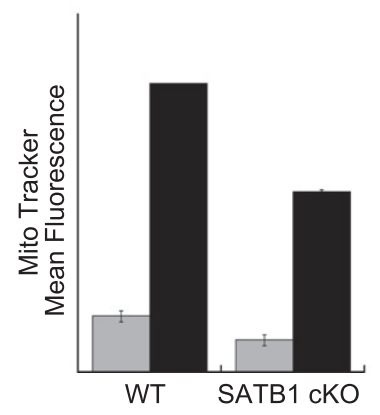

Figure 2. SATB1-TFAM axis is required for mitochondrial homeostasis.

(A) CD4 T cells were suspended in ATP assay buffer and incubated for $1 \mathrm{~min}$ at RT. Light intensity was indicative of intracellular ATP content (Lu-ATP). (B) Oxidative phosphorylation complex I enzyme activity was measured. Lysates were incubated on the plate for $3 \mathrm{~h}$ to capture complex I. Enzyme activity was determined by $\mathrm{NADH}$ oxidation to NAD+ and simultaneous dye reduction. Dye absorbance was measured with a plate reader at $450 \mathrm{~nm}$. (C) Levels of mtROS in CD4 T cells from WT, and SATB1CKO mice in the control and after TCR activation were measured by DCFDA staining and flow cytometry. (D) Quantification of mean fluorescence of DCFDA. (E) Representative MitoTracker staining histograms. WT and SATB1CKO T cells in the control and after TCR activation were stained with MitoTracker and analyzed by a flow cytometer. (F) Quantification of the mean fluorescence of MitoTracker. ${ }^{*} P<0.01$ versus WT. $N=5$. Data are shown as the means \pm SD. no change in the DCFDA-positive population was observed in SATB1CKO T cells after TCR stimulation (Fig 2C and D), demonstrating that TCR activation promotes mtROS production in WT but not in SATB1-deficient T cells. CD4 T cells were also incubated with MitoTracker. Similar to DCFDA staining, WT CD4 T cells after TCR activation increased the proportion of MitoTracker-positive cells. In contrast, the MitoTracker-positive population was less in SATB1cKO T cells after TCR stimulation (Fig 2E and F). Therefore, SATB1 is vital for proper mitochondrial function and necessary for mtROS production in T cells even in the resting state.

\section{SATB1-deficient T cells show high SHP-1 activity}

mtROS inactivates receptor-mediated signaling molecules such as phosphatases by oxidization, thereby enhancing and stabilizing kinase cascades (Meng et al, 2002; Kwon et al, 2004; Persson et al, 2004; Crump et al, 2012). As mitochondria localize near the TCR, mtROS may influence the TCR cascade. To determine whether mtROS oxidize phosphatases in TCR cascades, we investigated the oxidization status of SHP-1. Oxidized SHP-1 was weakly detected under basal conditions ( $0 \mathrm{~min}$ ) and clearly observed after TCR cross-linking (30 min) in naive CD4 T cells from WT mice (Fig 3A and B). In contrast, SATB1CKO T cells showed reduced oxidative SHP-1 modification under both resting and stimulated conditions (Fig $3 \mathrm{~A}$ and $B$ ). Next, to clarify the relationship between oxidation and phosphatase activity in SHP-1, we examined SHP-1 phosphatase activity in WT and SATB1CKO T cells before and after TCR stimulation. WT T cells showed low activity in the absence of TCR stimulation and gradual increases in the phosphatase activity at 60 and $120 \mathrm{~min}$ after TCR cross-linking (Fig 3C). In contrast, SATB1CKO T cells exhibited consistently high SHP-1 activity in both the absence and presence of TCR cross-linking (Fig 3C). These results suggest that oxidation inhibits SHP-1 phosphatase activity. To further explore this issue, we treated T-cell lysates with $\mathrm{H}_{2} \mathrm{O}_{2}$ and investigated whether mtROS-mediated oxidation suppresses SHP-1 function. SHP-1 in the cell lysates was oxidized by treatment with $\mathrm{H}_{2} \mathrm{O}_{2}$ (Fig 3D and E). As predicted, phosphatase activity in SHP-1 derived from SATB1CKO T cells was significantly decreased by oxidation with $\mathrm{H}_{2} \mathrm{O}_{2}$ 
(A)

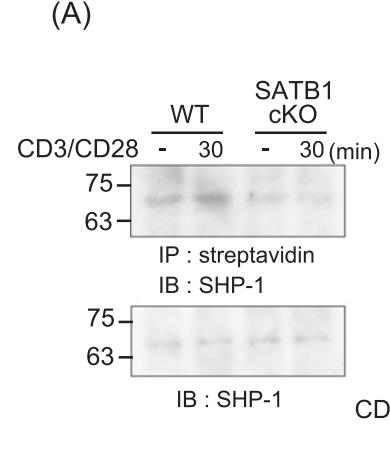

(D)

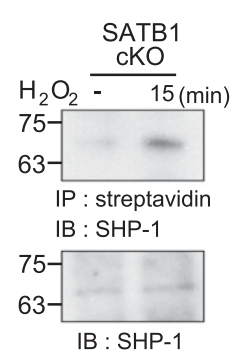

(B)

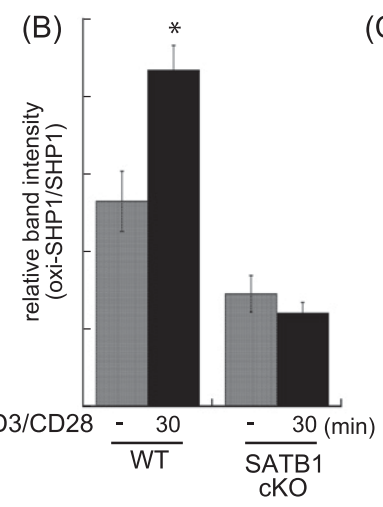

(E)

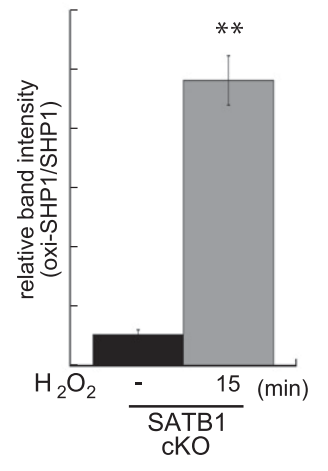

(C)

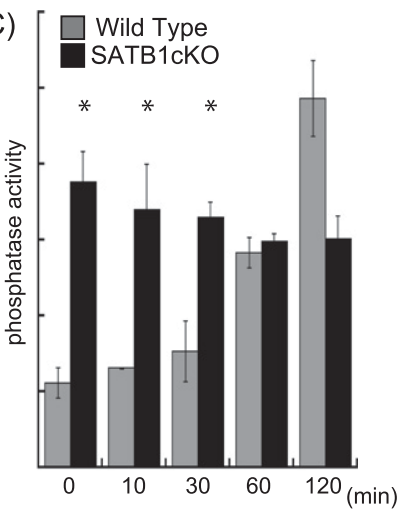

(F)

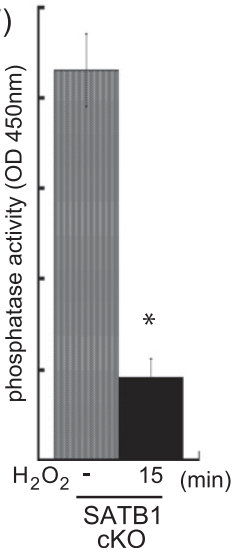

Figure 3. Phosphatase SHP-1 is regulated by mtROS. WT and SATB1CKO naïve CD4 T cells were stimulated with anti-CD3 and anti-CD28 antibodies for the indicated times and lysed in sample buffer. Oxidized proteins in the cell lysates were labeled with iodoaceto-PEG biotin and immunoprecipitated (A) Blots were probed for anti-SHP-1 antibody. (B) Band intensities were quantified with ImageJ v. 1.49. (C) Naïve CD4 T Cells were incubated for 0, 10, 20, 30, 60, and 120 min with anti-CD3 and anti-CD28 antibodies and lysed in phosphatase assay buffer. Phosphatase activity was assayed by incubation with PY containing peptide and liberated phosphate was measured by malachite green absorption at $450 \mathrm{~nm}$. (D, E) Lysates of SATB1-deficient naive CD4 T cells were incubated in the absence or presence of $\mathrm{H}_{2} \mathrm{O}_{2}$ for $15 \mathrm{~min}$. Oxidized SHP-1 was detected by immunoblotting with antibody against SHP-1 (D). (E) Band intensities were quantified with ImageJ v. 1.49 (E). (B, F) Phosphatase activity in the presence or absence of $\mathrm{H}_{2} \mathrm{O}_{2}$ was measured in (B). Data are shown as the means of three experiments performed in duplicate. ${ }^{*} P<0.01$ versus $W T$. ${ }^{* *} P<0.05$ versus WT. $N=5$. Data are shown as the means \pm SD. treatment (Fig 3F). These results indicate that SHP-1 in T cells is constitutively activated in the absence of SATB1 because of the lack of oxidation due to inability of mtROS generation. The results also suggest that SHP-1 oxidation is necessary for suppressing SHP-1 activity, which requires proper triggering of signaling cascades upon TCR stimulation.

\section{SATB1-deficient T cells have defective mitochondrial maintenance}

SATB1CKO T cells showed reduced ATP and mtROS production (Fig 2). Thus, we hypothesized that mitochondria in SATB1CKO $T$ cells are dysfunctional. To address this issue, we explored whether SATB1 deficiency in T cells decreases the mitochondrial content. We enumerated the mitochondria by staining them with MitoTracker. FACS-based staining intensity measurement and confocal imaging revealed fewer mitochondria in SATB1CKO T cells than in WT T cells (Figs 4A and B and S4A-D). Moreover, a drastic reduction in mitochondrial DNA confirmed that SATB1CKO T cells had relatively fewer mitochondria (Fig 4C).

TFAM plays a role in the maintenance of mitochondrial DNA and homeostasis (Ekstrand et al, 2004; Xu et al, 2009). Thus, we hypothesized that a relationship might exist between SATB1 and TFAM. We evaluated the level of TFAM using an anti-TFAM antibody. Compared with WT CD4 T cells, SATB1-deficient T cells expressed lower levels of TFAM protein (Fig 4D). Similar results were observed at the transcription level (Fig S5). These results suggest that SATB1 regulates TFAM expression.
T cells with disrupted TFAM expression presented with defective mitochondria (Fig 4A) and mitochondrial DNA (Figs $4 \mathrm{C}$ and S4). Therefore, down-regulated TFAM may cause defects in mitochondrial function and a loss of T-cell activity in SATB1CKO T cells. To test this hypothesis, we stimulated CD4 T cells from WT and TFAMcKO mice with anti-CD3 and anti-CD28 antibodies and analyzed the cell lysates by immunoblotting. ZAP70 phosphorylation was comparatively reduced in TFAMCKO T cells (Fig 4E). These results indicate that the quiescent phenotype of TFAMcKO $T$ cells resembles that of SATB1-deficient $T$ cells and that TFAM contributes to T-cell activation.

\section{Ectopic TFAM expression rescues defective TCR signaling in SATB1-deficient $T$ cells}

To further address whether TFAM expression complements SATB1 deficiency in T cells, we generated SATB1cKO mice with an ectopic TFAM gene. Transgenic human TFAM expression under SATB1sufficient or SATB1-deficient conditions was detected as similar protein levels (Fig 5A). The mitochondrial mass and DNA levels in T cells from SATB1CKO-TFAM transgenic (Tg) mice were restored nearly to those in WT T cells (Figs 4C, 5B and C, and S4). Decreased mtROS production in the steady state was also restored, and DCFDA fluorescence-positive population induced by TCR stimulation was rescued in T cells from SATB1CKO-TFAMTg mice (Fig 5D and E).

Active ZAP70 induced by TCR cross-linking was reconstituted to nearly normal levels in CD4 T cells from SATB1cKO-TFAMTg mice 
(A)

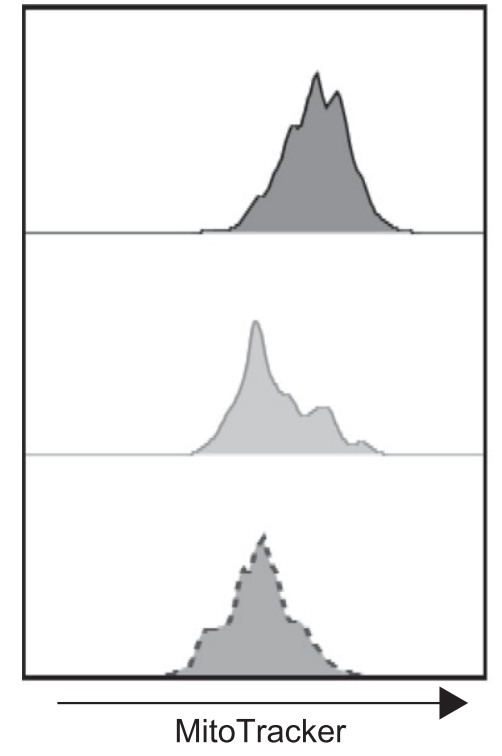

(C)

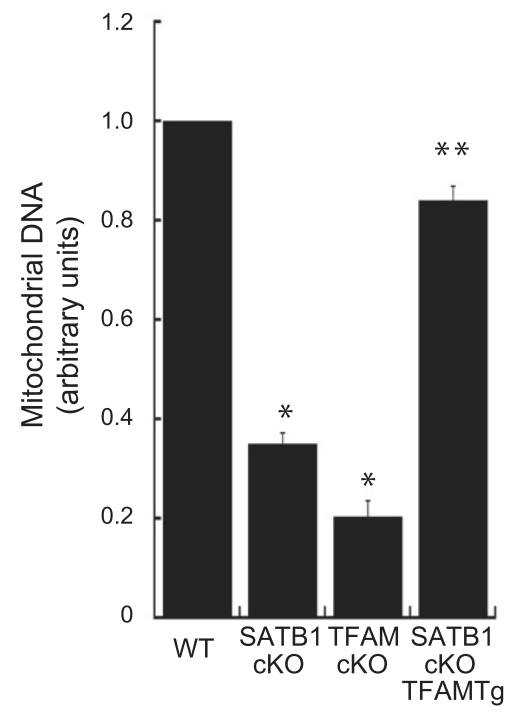

(B)

WT

CD4 Tcells

SATB1CKO

CD4 Tcells

TFAMCKO

CD4 Tcells

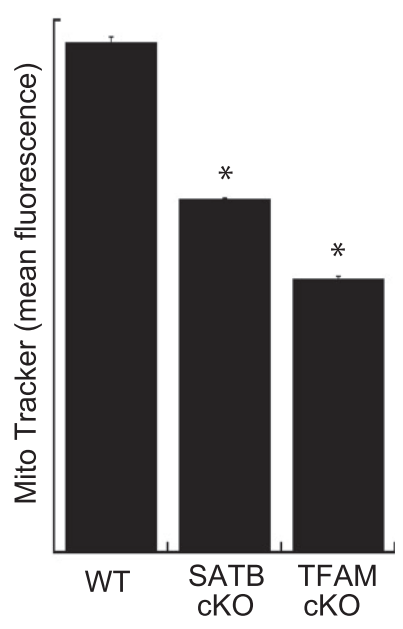

Figure 4. Loss of TFAM expression reduces T-cell activity.

(A) Representative MitoTracker staining histograms. WT and SATB1CKO T cells were stained with MitoTracker and analyzed by FACS. (B) Quantification of the mean fluorescence of MitoTracker is shown. (C) Mitochondrial DNA copy numbers in CD4 T cells from WT, SATB1 CKO, TFAMCKO, and SATB1CKO-TFAMTg mice were measured by RT-qPCR. The mitochondrial DNA level was normalized to the nuclear DNA level in each sample. The normalized index is indicated as an arbitrary unit $(W T=1)$. (D) TFAM and $\beta$-actin levels in CD4 T-cell lysates were analyzed by immunoblotting using the respective antibodies. (E) CD4 T cells were prepared from WT and TFAMCKO mice, stimulated for the indicated times with anti-CD3 and anti-CD28 antibodies, lysed, analyzed by SDS-PAGE, and immunoblotted with antibodies against phospho-ZAP70, and ZAP70. Similar results were obtained for three independent experiments. ${ }^{*} P<0.01$ versus WT. ${ }^{* *} P<0.01$ versus SATB1CKO T cells. $N=5$. Data are shown as the means \pm SD.

(D)

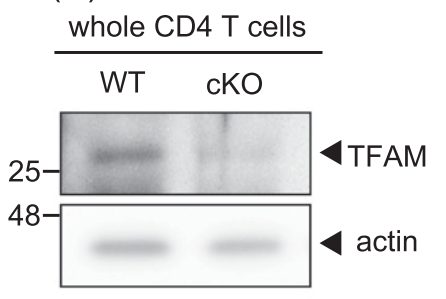

(E)

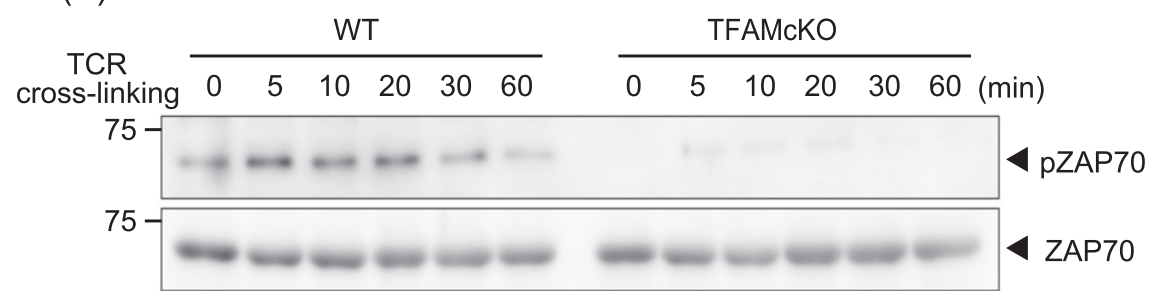

(Fig 5F). In addition, the mTOR and p70S6 kinase phosphorylation patterns induced by TCR stimulation were recovered (Figs 5G and S2). The AMPK phosphorylation observed in T cells lacking SATB1 expression was absent in T cells from SATB1CKO-TFAMTg mice in the resting state and induced upon TCR stimulation (Fig 5G). However, the level of phosphorylation in SATB1CKO-TFAMTg T cells was lower than WT T cells, suggesting that other SATB1-target genes than TFAM might be involved in proper AMPK phosphorylation pathway. Nevertheless, these results suggest that defective T-cell response in the absence of SATB1 might be due to lack of TFAM expression.

Finally, we examined whether ectopic TFAM expression could recover impaired T-cell function in SATB1CKO mice in vivo by the 
(A)

WT SATB1 cKO CD4 T cells CD4 T cells
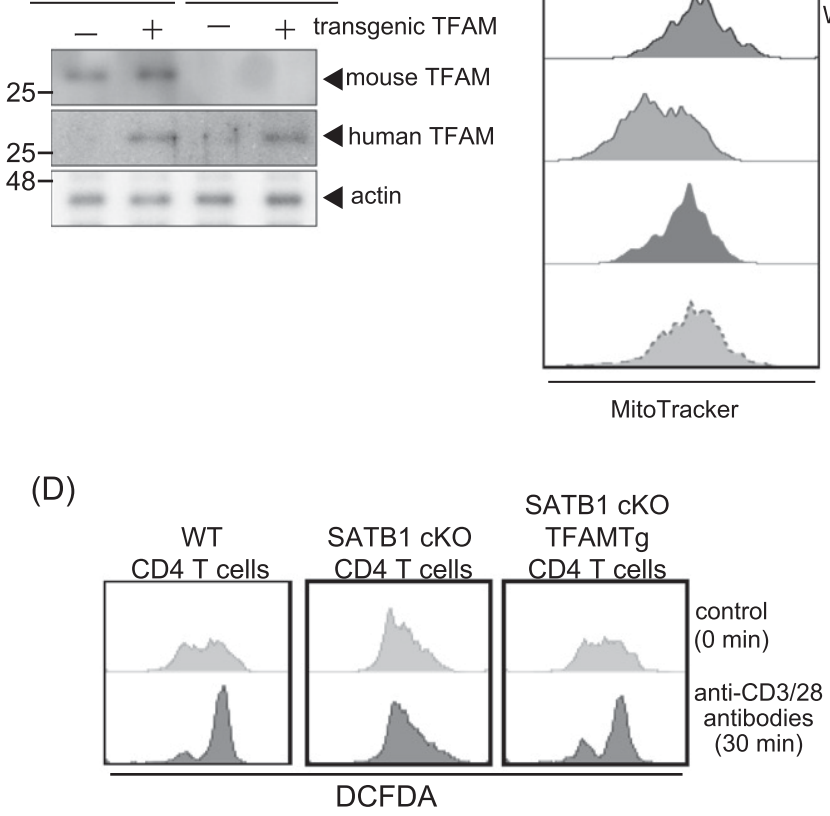

(B)

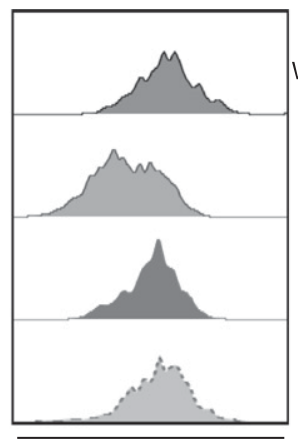

MitoTracker
(C)

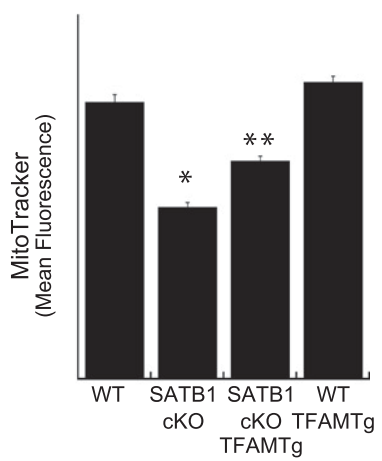

(E)

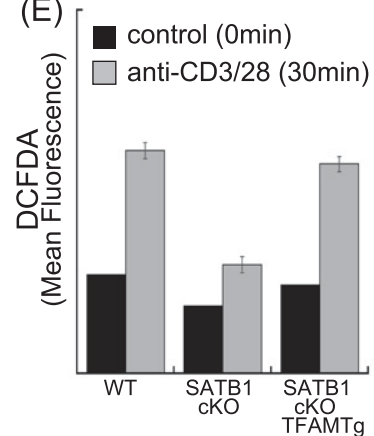

SATB CKO

TFAMTg

$(\mathrm{F})$

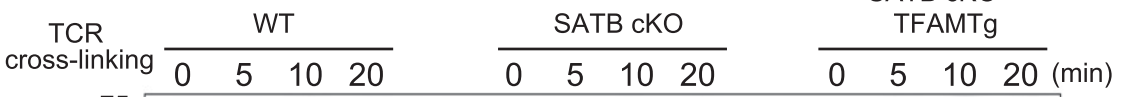

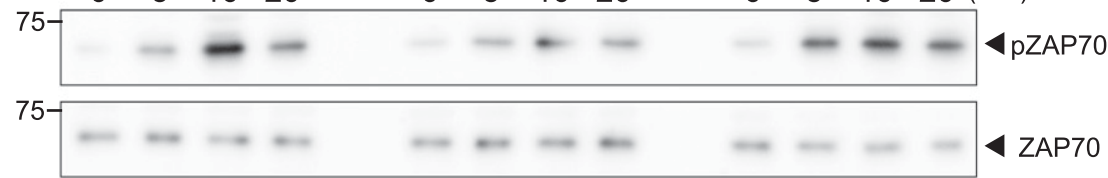

(G)

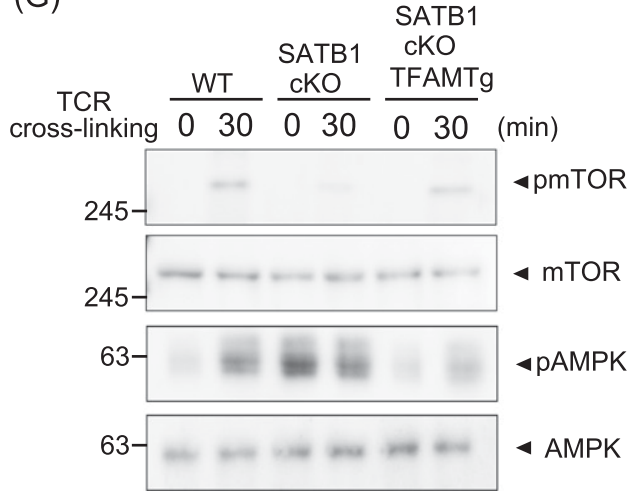

$(\mathrm{H})_{0.3}$

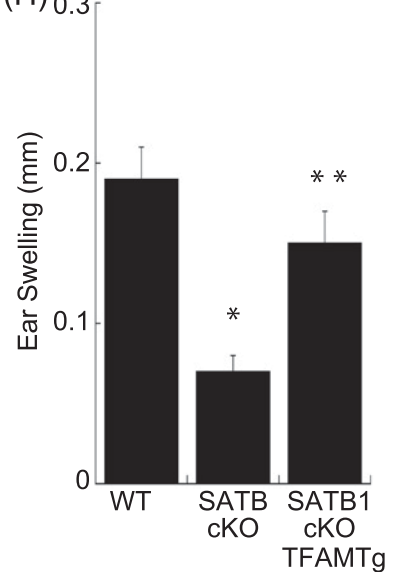

Figure 5. TFAM expression rescues T-cell activity in SATB1cKO mice.

(A) Immunoblot analysis of transgenic TFAM and endogenous TFAM in SATB1-sufficient or SATB1-deficinet CD4 T cells. (B) Representative MitoTracker staining histograms. WT, SATB1CKO, SATB1CKO-TFAMTg, and WT-TFAMTg T cells were stained with MitoTracker and analyzed by FACS. (C) Quantification of the mean fluorescence of MitoTracker. ${ }^{*} P<0.01$ versus WT. ${ }^{*} P<0.01$ versus SATB1CKO T cells. $N=5$. Data are shown as the means \pm SD. (D) ROS levels in CD4 T cells from WT, SATB1CKO, and SATB1CKOTFAMTg mice in control and TCR activation treatments were measured by DCFDA staining and flow cytometry. (E) Quantification of the mean fluorescence of DCFDA. $(\mathbf{F}, \mathbf{G})$ CD4 T cells were prepared from WT, SATB1CKO, and SATB1CKO-TFAMTg mice and functionally analyzed. T cells were stimulated with anti-CD3 and anti-CD28 
picryl chloride $(\mathrm{PCl})$ contact dermatitis model. This inflammatory skin disease is mediated by hapten specific T cells (Asherson \& Ptak, 1968; Austrup et al, 1997; Tietz et al, 1998). SATB1cKO mice showed less ear swelling compared with WT mice after re-challenged with PCI (Fig $5 \mathrm{H})$. In contrast, the contact hypersensitive response was rescued in SATB1CKO mice with exogenous TFAM expression. This result indicates that the T-cell response was intact in SATB1CKO-TFAMTg mice. Taken together, our results demonstrate that SATB1 plays a significant role in T-cell activation by maintaining mitochondria.

\section{Discussion}

In this study, we demonstrated that SATB1CKO T cells failed to express TFAM, maintain the mitochondria, and respond to TCR stimuli. Enforced TFAM expression rescued the activation capability of SATB1deficient T cells in response to TCR stimuli, indicating that lack of TFAM expression, which leads to loss of mitochondria contents, is largely responsible for T-cell dysfunction in SATB1CKO mice. Based on these findings, it seems that the role of SATB1 in T-cell activation is to maintain the mitochondria through TFAM expression in T cells.

TCR stimulation induces activation of SHP-1 by phosphorylation at its tyrosine residues (Zhang et al, 2000). As we showed in this study, SATB1CKO T cells possess high SHP-1 activity, even in the resting state. In addition, tyrosine phosphorylation levels of signal molecules proximate to TCR were extremely low. Therefore, SHP-1 activation is enhanced in SATB1-deficient $T$ cells by other mechanisms than phosphorylation. SATB1-deficient T cells showed a smaller number of mitochondria and lower production of ATP than in WT T cells. The metabolic regulator AMPK was also activated in SATB1-deficient T cells in the resting state. These results suggest that AMPK-mediated machinery may activate SHP-1 in SATB1-deficient T cells. AMPK activation also suggested that metabolic dysfunction (low amount of ATP) in SATB1-deficient T cells. As cellular ATP is necessary for T-cell activation (van der Windt et al, 2012; Pearce et al, 2013; Maciolek et al, 2014), metabolic stress interferes to induce T-cell activation in SATB1deficient T cells. We should note that these results are not sufficient to determine either AMPK-mediated SHP-1 activation or energy stress is the causative reason of T-cell dysfunction in SATB1-deficient T cells.

SATB1 is a molecular adapter for chromatin-remodeling complexes that tighten or loosen chromosomal DNA into an active or inactive state (Yasui et al, 2002). SATB1 forms a unique transcriptional chromatin structure and up-regulates genes of Th2 cytokines (Cai et al, 2006). As SATB1 regulates the activity of genes near its DNA-binding sites, we used a ChIP assay with anti-SATB1 antibodies to detect SATB1 binding to the regulatory regions of Tfam. However, there was no evidence that SATB1 binds to the Tfam gene (data not shown). Tfam expression is regulated by various transcription factors such as PGC-1 $\alpha$ and NRF (Piantadosi \& Suliman, 2006; Kozhukhar \& Alexeyev, 2019). Thus, SATB1 may indirectly up-regulate the transcriptional activity of Tfam. SATB1 may also associate with various proteins, such as $\beta$-catenin and up-regulate genes in activated T cells (Notani et al, 2010). Thus, identification of the SATB1 binding partner may reveal the regulatory mechanisms of Tfam expression.

Mitochondrial activities are known to be linked to T-cell functions (Bantug et al, 2018; Desdin-Mico et al, 2018; Zhu \& Thompson, 2019). T cells sense extracellular chemotactic factor gradients that recruit them to infection or inflammatory sites and cause various changes in T-cell morphology (Campello et al, 2006). The cell shape change indicates polarization that initiates the formation of leading edges and uropods. Mitochondria accumulate at uropods and provide ATP during directed T-cell migration (Campello \& Scorrano, 2010). After clearing invaded pathogens, most effector $T$ cells diminish during the contraction phase, whereas memory $T$ cells employ their activities such as oxidation lipid metabolism (Bantug et al, 2018; Desdin-Mico et al, 2018; Zhu \& Thompson, 2019). Memory T cells display an increased mitochondrial number and maximal mitochondrial respiratory capacity (Pearce et al, 2009; van der Windt et al, 2012). Therefore, SATB1 may be involved in maintaining effector T-cell potential and supporting mitochondrial activity.

Here, we demonstrated that SATB1 modulates TCR signaling. To the best of our knowledge, this is the first study to demonstrate that SATB1-mediated mtROS production is required to generate T-cell responses. mtROS from mitochondria regulated by SATB1 maintain ZAP70 and ERK activation downstream of TCR via oxidative SHP-1 inhibition. Therefore, SATB1 is a key molecule in mitochondrial activity and is essential for T-cell homeostasis.

\section{Materials and Methods}

\section{Mice}

SATB1-floxed mice and transgenic mice expressing mitochondrial transcription factor A (TFAM) were previously described (Ikeuchi et al, 2005; Kondo et al, 2016). Vav-Cre and TFAM-floxed mice were purchased from The Jackson Laboratory. Lck-Cre mice were obtained from the Laboratory Animal Resource Bank at NIBIOHN. SATB1 or TFAM conditional knockout mice were generated by crossing floxed, Vav-Cre, and Lck-Cre mice. All mice were maintained on a C57BL/ 6 background under specific pathogenfree conditions at the Toho University School of Medicine animal facility (Kuwabara et al, 2009). All mouse experiments were approved by the Toho University Animal Care and User Committee (No. 20-51-435) and Toho University Safety Committee for Recombinant DNA Experiments (No. 20-51-440). Experiments were performed on 8- to 12-wk-old mice.

\section{Antibodies}

Anti-SHP-1 was purchased from Abcam. Anti-AMPK, antiphosphorylated-AMPK, anti-ZAP70, anti-phosphorylated-ZAP70, 
anti-mTOR, anti-phosphorylated-mTOR, anti-p70 S6 kinase, and antiphosphorylated-p70 S6 kinase antibodies were acquired from Cell Signaling Technology.

\section{Mitochondrial DNA}

qPCR quantified the mitochondrial DNA copy number. Total DNA was isolated with a DNeasy blood and tissue kit (QIAGEN GmbH) according to the manufacturer's instructions. Mitochondrially encoded nicotinamide adenine dinucleotide NADH dehydrogenase 1 (mND1) and hexokinase gene 2 (NK2) DNA were amplified with Maxima SYBR Green qPCR Master Mix (Thermo Fisher Scientific). To quantify the mitochondrial DNA copy number, the ratio of mitochondrial ND1 to nuclear HK2 was calculated by the $2^{-\triangle \Delta C t}$ method. The primers used were forward $5^{\prime}$-CTAGCAGAAACAAACCGGGC-3' and reverse 5'-CCGGCTGCGTATTCTACGTT-3' for ND1 and forward 5'GCCAGCCTCTCCTGATTTTAGTGT-3' and reverse 5'-GGGAACACAAAAGACCTCTTCTGG-3' for HK2.

\section{Cell imaging}

CD4 T cells from the spleen were prepared by cell sorting and incubated in RPMI-1640 medium supplemented with 10\% (vol/vol) FCS and $50 \mu \mathrm{M}$ of 2-ME for 60 min followed by addition of MitoTracker (Invitrogen) for $20 \mathrm{~min}$. The cells were washed with ice-cold PBS and fixed with $4 \%$ (vol/vol) PFA in PBS for $10 \mathrm{~min}$ at $25^{\circ} \mathrm{C}$. The nuclei were stained with $1 \mu \mathrm{M}$ TO-PRO3 (Invitrogen) for $20 \mathrm{~min}$ at $25^{\circ} \mathrm{C}$. The cells were observed under a confocal laser-scanning microscope (LSM510; Carl Zeiss AG). Fluorescence images were processed with Adobe Photoshop (Adobe Systems, Inc.).

\section{Contact hypersensitivity response}

Mice were epicutaneously sensitized on their shaved abdomens with $150 \mu \mathrm{l}$ of $7 \%$ ( $\mathrm{vol} / \mathrm{vol}$ ) 2,4,5-trititrochlorobenzene (picryl chloride [PCl]; NacalaiTesque, Kyoto, Japan) in 4:1 (vol/vol) acetone/ olive oil. $6 \mathrm{~d}$ after sensitization, the right ears were challenged with $20 \mu \mathrm{l}$ of $1 \%(\mathrm{vol} / \mathrm{vol}) \mathrm{PCl}$ in the vehicle. The left ears were treated with vehicle alone. After $24 \mathrm{~h}$, ear thickness was measured with a dial thickness gauge (Dial Caliper, Mitutoyo). Ear thickness was calculated according to the following formula: ear thickness = (ear thickness after $24 \mathrm{~h}$ elicitation; right ear) - (control ear thickness; left ear).

\section{Respiratory enzyme activity}

Mitochondrial complex I-driven respiratory capacity was measured in murine CD4 T cells from WT and SATB1CKO mice. Specific rotenone-sensitive NADH oxidoreductase activity was measured with a Complex I enzyme activity microplate assay kit (Abcam) according to the manufacturer's instructions.

\section{Immunoblotting}

Immunoblotting was performed as previously described (Kuwabara et al, 2012; Matsui et al, 2018). T cells were incubated at $37^{\circ} \mathrm{C}$ for $4 \mathrm{~h}$ in FCS-free RPMI-1640 for cell cycle synchronization. The cells were washed and incubated at a density of $2.0 \times 10^{6} / \mathrm{ml}$ and stimulated with plate-bound anti-CD3 and anti-CD28 antibodies for the times indicated in Figs and Figs 13 -Figs 15. Cell extracts were generated from cultured cells with extraction buffer (50 mM Tris- $\mathrm{HCl}$ [pH 7.4], $1 \%$ [vol/vol] Triton X-100, $450 \mathrm{mM} \mathrm{NaCl}, 1 \mathrm{mM}$ EDTA, and $1 \mathrm{mM}$ DTT) and proteinase inhibitors. Lysates were centrifuged at $12,000 \mathrm{~g}$ for 10 $\min$ at $4^{\circ} \mathrm{C}$. Protein concentrations in the supernatants were determined by bicinchoninic acid (Thermo Fisher Scientific) protein assay. Samples were suspended in $2 \times$ sample buffer $(75 \mathrm{mM}$ Tris-HCl [pH 6.8], 10\% [vol/vol] glycerol, 2\% [vol/vol] SDS, 0.05\% [wt/ vol] bromophenol blue, and 2.5\% [vol/vol] 2-ME). They were then loaded onto $7.5 \%$ (wt/vol) or $10 \%$ (wt/vol) SDS-PAGE gels and transferred to polyvinylidene difluoride filters in transfer buffer (25 mM Tris, 0.192 M glycine, and 20\% [vol/vol] methanol). The filters were then blocked with $1 \%(\mathrm{vol} / \mathrm{vol}) \mathrm{BSA}$ in Tris-buffered saline containing $0.05 \%$ (vol/vol) Tween 20 at 25 degrees for $2 \mathrm{~h}$, incubated with the indicated antibodies, incubated with anti-mouse IgG or anti-rabbit IgG coupled with HRP, and visualized with an enhance chemiluminescence detection system (GE Healthcare). Quantitation was performed with ImageJ v. 1.49 software (National Institute of Health).

\section{Flow cytometry}

Antibodies used for flow cytometry [CD4(GK1.5), CD8(53-6.7), B220(RA3-6B2), CD44(IM7), and CD62L(MEL-14)] were purchased from BioLegend. Erythrocytes were depleted from a single-cell spleen and lymph node suspension, incubated with a mixture of fluorescence-conjugated antibodies at $4^{\circ} \mathrm{C}$ for $30 \mathrm{~min}$, and washed three times with FACS buffer (1\% [vol/vol] FCS in PBS and 0.05\% [wt/ vol] $\left.\mathrm{NaN}_{3}\right) \cdot \mathrm{CD}^{+} \mathrm{CD}_{22} \mathrm{~L}^{\text {hi }} \mathrm{CD} 44^{\mathrm{lo}} \mathrm{T}$ cells were then enriched with the cell sorter FACSAria III (BD Biosciences).

Mitochondrial mass and cellular ROS were assessed with MitoTracker (Invitrogen) and a DCFDA cellular ROS detection assay kit (Abcam), respectively, according to the manufacturers' instructions. Flow cytometry analysis of the cell cycle was performed with a BrdU flow kit (BD Biosciences). To assess viability, the cells were stained with 7-amino-actinomycin (BioLegend) according to the manufacturer's instructions. Data were acquired with the LSR Fortessa X-20 flow cytometer (BD BiosciencesA) and analyzed with Tree Star FlowJo version 10.5.3 (FlowJo LLC).

\section{Detection of oxidized proteins}

Cells were washed with ice-cold PBS and lysed in oxidized lysis buffer (200 mM ammonium bicarbonate [pH 8.0] and 0.1\% [vol/vol] SDS). Cell lysates were incubated under anaerobic conditions. Free thiols were masked with $20 \mathrm{mM}$ iodoacetoamide. Oxidized thiols were reduced with $4 \mathrm{mM}$ DTT and labeled with $1 \mathrm{mM}$ iodoaceto-PEG biotin. Biotinylated proteins were detected by immunoblot analysis.

\section{Phosphotyrosine phosphatase assay}

T-cell extracts (50 $\mu \mathrm{g}$ protein) were incubated with $3 \mu \mathrm{g}$ anti-SHP-1 antibody for $6 \mathrm{~h}$ at $4^{\circ} \mathrm{C}$ in a total volume of $100 \mu \mathrm{l}$. Protein-A beads were added to each sample and incubated for $4 \mathrm{~h}$ at $4^{\circ} \mathrm{C}$ with gentle rotation. Samples were centrifuged and washed 6× with $10 \mathrm{mM}$ Tris $-\mathrm{HCl}$ 
buffer ( $\mathrm{pH}$ 7.4) containing $100 \mathrm{mM}$ DTT. Washed beads were resuspended in $25 \mu \mathrm{l}$ of $10 \mathrm{mM}$ Tris- $\mathrm{HCl}$ buffer ( $\mathrm{pH} 7.4$ ) and incubated with $100 \mathrm{mM}$ tyrosine phosphopeptide $\mathrm{NH}_{2}$-RRLIEDAEPYAARG-COOH for 30 min at $25^{\circ} \mathrm{C}$ using reagents obtained from Upstate Biotechnology. The reaction was terminated with $25^{\circ} \mathrm{C}$ malachite green solution and incubated for 15 min to allow for color development. Phosphatase activity was measured at $620 \mathrm{~nm}$ in a microtiter plate reader (iMark; Bio-Rad Laboratories).

\section{Supplementary Information}

Supplementary Information is available at https://doi.org/10.26508/lsa. 202101093

\section{Acknowledgements}

This work was supported by a grant from Takeda Science Foundation to T Kuwabara, the Japan Society for the Promotion of Science Grant-in-Aid for Scientific Research (GrantNo. 17K08892 to T Kuwabara and No. 19 K07613 to M Kondo), a Toho University Grant for Research Initiative Program (TU-GRIP), and a Grant-in-Aid for the Private University Research Branding Project from the Ministry of Education, Culture, Sports Science and Technology to M Kondo. The authors thank Editage (www.editage.jp) for English language editing.

\section{Author Contributions}

T Kuwabara: project administration and writing-original draft, review, and editing.

F Ishikawa: supervision.

M Ikeda: supervision.

T Ide: supervision.

T Kohwi-Shigematsu: supervision.

Y Tanaka: supervision.

M Kondo: supervision and project administration.

\section{Conflict of Interest Statement}

The authors declare that they have no conflict of interest.

\section{References}

Akiba Y, Kuwabara T, Mukozu T, Mikami T, Kondo M (2018) Special AT-rich sequence binding protein 1 is required for maintenance of $T$ cell receptor responsiveness and development of experimental autoimmune encephalomyelitis. Microbiol Immunol 62: 255-268. doi:10.1111/1348-0421.12579

Arciuch VGA, Elguero ME, Poderoso JJ, Carreras MC (2012) Mitochondrial regulation of cell cycle and proliferation. Antioxid Redox Signal 16: 1150-1180. doi:10.1089/ars.2011.4085

Asherson GL, Ptak W (1968) Contact and delayed hypersensitivity in the mouse. I. Active sensitization and passive transfer. Immunology 15 405-416.

Austrup F, Vestweber D, Borges E, Löhning M, Bräuer R, Herz U, Renz H, Hallmann R, Scheffold A, Radbruch A, et al (1997) P- and E-selectin mediate recruitment of T-helper-1 but not T-helper-2 cells into inflammed tissues. Nature 385: 81-83. doi:10.1038/385081a0

Baixauli F, Martín-Cófreces NB, Morlino G, Carrasco YR, Calabia-Linares C, Veiga E, Serrador JM, Sánchez-Madrid F (2011) The mitochondrial fission factor dynamin-related protein 1 modulates T-cell receptor signalling at the immune synapse. EMBO / 30: 1238-1250. doi:10.1038/ emboj.2011.25

Banan M, Rojas IC, Lee WH, King HL, Harriss JV, Kobayashi R, Webb CF, Gottlieb PD (1997) Interaction of the nuclear matrix-associated region (MAR)binding proteins, SATB1 and CDP/Cux, with a MAR element (L2a) in an upstream regulatory region of the mouse CD8a gene. J Biol Chem 272: 18440-18452. doi:10.1074/jbc.272.29.18440

Bantug GR, Galluzzi L, Kroemer G, Hess C (2018) The spectrum of T cell metabolism in health and disease. Nat Rev Immunol 18: 19-34. doi:10.1038/nri.2017.99

Cai S, Han HJ, Kohwi-Shigematsu T (2003) Tissue-specific nuclear architecture and gene expression regulated by SATB1. Nat Genet 34: 42-51. doi:10.1038/ng1146

Cai S, Lee CC, Kohwi-Shigematsu T (2006) SATB1 packages densely looped, transcriptionally active chromatin for coordinated expression of cytokine genes. Nat Genet 38: 1278-1288. doi:10.1038/ng1913

Campello S, Lacalle RA, Bettella M, Mañes S, Scorrano L, Viola A (2006) Orchestration of lymphocyte chemotaxis by mitochondrial dynamics. J Exp Med 203: 2879-2886. doi:10.1084/jem.20061877

Campello S, Scorrano L (2010) Mitochondrial shape changes: Orchestrating cell pathophysiology. EMBO Rep 11: 678-684. doi:10.1038/ embor.2010.115

Capasso M, Bhamrah MK, Henley T, Boyd RS, Langlais C, Cain K, Dinsdale D, Pulford K, Khan M, Musset B, et al (2010) HVCN1 modulates BCR signal strength via regulation of $B C R$-dependent generation of reactive oxygen species. Nat Immunol 11: 265-272. doi:10.1038/ni.1843

Carter JD, Neel BG, Lorenz U (1999) The tyrosine phosphatase SHP-1 influences thymocyte selection by setting TCR signaling thresholds. Int Immunol 11: 1999-2014. doi:10.1093/intimm/11.12.1999

Chandel NS (2010) Mitochondrial regulation of oxygen sensing. Adv Exp Med Biol 661: 339-354. doi:10.1007/978-1-60761-500-2_22

Cockerill PN, Yuen MH, Garrard WT (1987) The enhancer of the immunoglobulin heavy chain locus is flanked by presumptive chromosomal loop anchorage elements. J Biol Chem 262: 5394-5397. doi:10.1016/s0021-9258(18)61200-1

Courtney AH, Lo WL, Weiss A (2018) TCR signaling: Mechanisms of initiation and propagation. Trends Biochem Sci 43: 108-123. doi:10.1016/ j.tibs.2017.11.008

Crump KE, Juneau DG, Poole LB, Haas KM, Grayson JM (2012) The reversible formation of cysteine sulfenic acid promotes B-cell activation and proliferation. Eur J Immunol 42: 2152-2164. doi:10.1002/eji.201142289

Desdín-Micó G, Soto-Heredero G, Mittelbrunn M (2018) Mitochondrial activity in T cells. Mitochondrion 41: 51-57. doi:10.1016/j.mito.2017.10.006

Dickinson LA, Joh T, Kohwi Y, Kohwi-Shigematsu T (1992) A tissue-specific MAR/SAR DNA-binding protein with unusual binding site recognition. Cell 70: 631-645. doi:10.1016/0092-8674(92)90432-c

Ekstrand MI, Falkenberg M, Rantanen A, Park CB, Gaspari M, Hultenby K, Rustin P, Gustafsson CM, Larsson NG (2004) Mitochondrial transcription factor A regulates mtDNA copy number in mammals Hum Mol Genet 13: 935-944. doi:10.1093/hmg/ddh109

Finkel T (2011) Signal transduction by reactive oxygen species. J Cell Biol 194 7-15. doi:10.1083/jcb.201102095

Fisher RP, Parisi MA, Clayton DA (1989) Flexible recognition of rapidly evolving promoter sequences by mitochondrial transcription factor 1. Genes Dev 3: 2202-2217. doi:10.1101/gad.3.12b.2202 
Gaud G, Lesourne R, Love PE (2018) Regulatory mechanisms in T cell receptor signalling. Nat Rev Immunol 18: 485-497. doi:10.1038/s41577018-0020-8

Gunter TE, Yule DI, Gunter KK, Eliseev RA, Salter JD (2004) Calcium and mitochondria. FEBS Lett 567: 96-102. doi:10.1016/j.febslet.2004.03.071

Ikeuchi M, Matsusaka H, Kang D, Matsushima S, Ide T, Kubota T, Fujiwara T, Hamasaki N, Takeshita A, Sunagawa K, et al (2005) Overexpression of mitochondrial transcription factor a ameliorates mitochondrial deficiencies and cardiac failure after myocardial infarction. Circulation 112: 683-690. doi:10.1161/CIRCULATIONAHA.104.524835

Kondo M, Tanaka Y, Kuwabara T, Naito T, Kohwi-Shigematsu T, Watanabe A (2016) SATB1 plays a critical role in establishment of immune tolerance. J Immunol 196: 563-572. doi:10.4049/jimmunol.1501429

Kosugi A, Sakakura J, Yasuda K, Ogata M, Hamaoka T (2001) Involvement of SHP-1 tyrosine phosphatase in TCR-mediated signaling pathways in lipid rafts. Immunity 14: 669-680. doi:10.1016/s1074-7613(01)00146-7

Kozhukhar N, Alexeyev MF (2019) Limited predictive value of TFAM in mitochondrial biogenesis. Mitochondrion 49: 156-165. doi:10.1016/ j.mito.2019.08.001

Kumar PP, Bischof O, Purbey PK, Notani D, Urlaub H, Dejean A, Galande S (2007) Functional interaction between PML and SATB1 regulates chromatin-loop architecture and transcription of the MHC class I locus. Nat Cell Biol 9: 45-56. doi:10.1038/ncb1516

Kuwabara T, Ishikawa F, Yasuda T, Aritomi K, Nakano H, Tanaka Y, Okada Y, Lipp M, Kakiuchi T (2009) CCR7 ligands are required for development of experimental autoimmune encephalomyelitis through generating IL-23-dependent Th17 cells. J Immunol 183: 2513-2521. doi:10.4049/ jimmunol.0800729

Kuwabara T, Tanaka Y, Ishikawa F, Kondo M, Sekiya H, Kakiuchi T (2012) CCR7 ligands up-regulate IL-23 through PI3-kinase and NF-K B pathway in dendritic cells. J Leukoc Biol 92: 309-318. doi:10.1189/jlb.0811415

Kwon J, Lee SR, Yang KS, Ahn Y, Kim YJ, Stadtman ER, Rhee SG (2004) Reversible oxidation and inactivation of the tumor suppressor PTEN in cells stimulated with peptide growth factors. Proc Natl Acad Sci U S A 101: 16419-16424. doi:10.1073/pnas.0407396101

Larsson NG, Wang J, Wilhelmsson H, Oldfors A, Rustin P, Lewandoski M, Barsh GS, Clayton DA (1998) Mitochondrial transcription factor A is necessary for mtDNA maintenance and embryogenesis in mice. Nat Genet 18: 231-236. doi:10.1038/ng0398-231

Lorenz U (2009) SHP-1 and SHP-2 in T cells: Two phosphatases functioning at many levels. Immunol Rev 228: 342-359. doi:10.1111/j.1600065X.2008.00760.X

Maciolek JA, Pasternak JA, Wilson HL (2014) Metabolism of activated T lymphocytes. Curr Opin Immunol 27: 60-74. doi:10.1016/ j.coi.2014.01.006

Maclver NJ, Michalek RD, Rathmell JC (2013) Metabolic regulation of T lymphocytes. Annu Rev Immunol 31: 259-283. doi:10.1146/annurevimmunol-032712-095956

Matsui Y, Kuwabara T, Eguchi T, Nakajima K, Kondo M (2018) Acetylation regulates the MKK4-JNK pathway in T cell receptor signaling. Immunol Lett 194: 21-28. doi:10.1016/j.imlet.2017.12.002

Meng TC, Fukada T, Tonks NK (2002) Reversible oxidation and inactivation of protein tyrosine phosphatases in vivo. Mol Cell 9:387-399. doi:10.1016/ s1097-2765(02)00445-8

Mills EL, Kelly B, O'Neill LAJ (2017) Mitochondria are the powerhouses of immunity. Nat Immunol 18: 488-498. doi:10.1038/ni.3704

Molina TJ, Kishihara K, Siderovski DP, van Ewijk W, Narendran A, Timms E, Wakeham A, Paige CJ, Hartmann KU, Veillette A, et al (1992) Profound block in thymocyte development in mice lacking p56lck. Nature 357: 161-164. doi:10.1038/357161a0

Nel AE (2002) T-cell activation through the antigen receptor. Part 1: Signaling components, signaling pathways, and signal integration at the T-cell antigen receptor synapse. J Allergy Clin Immunol 109: 758-770. doi:10.1067/mai.2002.124259

Nel AE, Slaughter N (2002) T-cell activation through the antigen receptor. Part 2: Role of signaling cascades in T-cell differentiation, anergy, immune senescence, and development of immunotherapy. J Allergy Clin Immunol 109: 901-915. doi:10.1067/mai.2002.124965

Nisoli E, Tonello C, Cardile A, Cozzi V, Bracale R, Tedesco L, Falcone S, Valerio A, Cantoni O, Clementi E, et al (2005) Calorie restriction promotes mitochondrial biogenesis by inducing the expression of eNOS Science 310: 314-317. doi:10.1126/science.1117728

Notani D, Gottimukkala KP, Jayani RS, Limaye AS, Damle MV, Mehta S, Purbey PK, Joseph J, Galande S (2010) Global regulator SATB1 recruits betacatenin and regulates $\mathrm{T}(\mathrm{H}) 2$ differentiation in Wnt-dependent manner. PLoS Biol 8: e1000296. doi:10.1371/journal.pbio.1000296

Pearce EL, Poffenberger MC, Chang CH, Jones RG (2013) Fueling immunity: Insights into metabolism and lymphocyte function. Science 342: 1242454. doi:10.1126/science.1242454

Pearce EL, Walsh MC, Cejas PJ, Harms GM, Shen H, Wang LS, Jones RG, Choi Y (2009) Enhancing CD8 T-cell memory by modulating fatty acid metabolism. Nature 460: 103-107. doi:10.1038/nature08097

Persson C, Sjöblom T, Groen A, Kappert K, Engström U, Hellman U, Heldin CH, den Hertog J, Ostman A (2004) Preferential oxidation of the second phosphatase domain of receptor-like PTP-alpha revealed by an antibody against oxidized protein tyrosine phosphatases. Proc Natl Acad Sci U S A 101: 1886-1891. doi:10.1073/pnas.0304403101

Phillips JE, Corces VG (2009) CTCF: Master weaver of the genome. Cell 137: 1194-1211. doi:10.1016/j.cell.2009.06.001

Piantadosi CA, Suliman HB (2006) Mitochondrial transcription factor A induction by redox activation of nuclear respiratory factor 1. J Biol Chem 281: 324-333. doi:10.1074/jbc.M508805200

Quintana A, Schwindling C, Wenning AS, Becherer U, Rettig J, Schwarz EC, Hoth $M$ (2007) T cell activation requires mitochondrial translocation to the immunological synapse. Proc Natl Acad Sci U S A 104: 14418-14423. doi:10.1073/pnas.0703126104

Schneider R, Grosschedl R (2007) Dynamics and interplay of nuclear architecture, genome organization, and gene expression. Genes Dev 21: 3027-3043. doi:10.1101/gad.1604607

Smith-Garvin JE, Koretzky GA, Jordan MS (2009) T cell activation. Annu Rev Immunol 27: 591-619. doi:10.1146/annurev.immunol.021908.132706

Stefanová I, Hemmer B, Vergelli M, Martin R, Biddison WE, Germain RN (2003) TCR ligand discrimination is enforced by competing ERK positive and SHP-1 negative feedback pathways. Nat Immunol 4: 248-254. doi:10.1038/ni895

Straus DB, Weiss A (1992) Genetic evidence for the involvement of the lck tyrosine kinase in signal transduction through the T cell antigen receptor. Cell 70: 585-593. doi:10.1016/0092-8674(92)90428-f

Tait SW, Green DR (2012) Mitochondria and cell signalling. J Cell Sci 125: 807-815. doi:10.1242/jcs.099234

Tanaka Y, Sotome T, Inoue A, Mukozu T, Kuwabara T, Mikami T, KowhiShigematsu T, Kondo M (2017) SATB1 conditional knockout results in Sjögren's syndrome in mice. J Immunol 199: 4016-4022. doi:10.4049/ jimmunol.1700550

Tietz W, Allemand Y, Borges E, von Laer D, Hallmann R, Vestweber D, Hamann A (1998) CD4+ T cells migrate into inflamed skin only if they express ligands for E- and P-selectin. J Immunol 161: 963-970.

van der Windt GJ, Everts B, Chang CH, Curtis JD, Freitas TC, Amiel E, Pearce EJ, Pearce EL (2012) Mitochondrial respiratory capacity is a critical regulator of CD8+ T cell memory development. Immunity 36: 68-78. doi:10.1016/j.immuni.2011.12.007

van Leeuwen JE, Samelson LE (1999) T cell antigen-receptor signal transduction. Curr Opin Immunol 11: 242-248. doi:10.1016/s09527915(99)80040-5 
Xu S, Zhong M, Zhang L, Wang Y, Zhou Z, Hao Y, Zhang W, Yang X, Wei A, Pei L, et al (2009) Overexpression of Tfam protects mitochondria against beta-amyloid-induced oxidative damage in SH-SY5Y cells. FEBS I 276: 3800-3809. doi:10.1111/j.1742-4658.2009.07094.X

Yasui D, Miyano M, Cai S, Varga-Weisz P, Kohwi-Shigematsu T (2002) SATB1 targets chromatin remodelling to regulate genes over long distances. Nature 419: 641-645. doi:10.1038/nature01084

Zhang J, Somani AK, Siminovitch KA (2000) Roles of the SHP-1 tyrosine phosphatase in the negative regulation of cell signalling. Semin Immunol 12: 361-378. doi:10.1006/smim.2000.0223

Zhang W, Sloan-Lancaster J, Kitchen J, Trible RP, Samelson LE (1998) LAT: The ZAP-70 tyrosine kinase substrate that links T cell receptor to cellular activation. Cell 92: 83-92. doi:10.1016/ s0092-8674(00)80901-0
Zhong XP, Carabaña J, Krangel MS (1999) Flanking nuclear matrix attachment regions synergize with the $T$ cell receptor delta enhancer to promote V(D)J recombination. Proc Natl Acad Sci U S A 96: 11970-11975. doi:10.1073/pnas.96.21.11970

Zhu J, Thompson CB (2019) Metabolic regulation of cell growth and proliferation. Nat Rev Mol Cell Biol 20: 436-450. doi:10.1038/s41580019-0123-5

Zlatanova JS, van Holde KE (1992) Chromatin loops and transcriptional regulation. Crit Rev Eukaryot Gene Expr 2: 211-224.

(c)

License: This article is available under a Creative Commons License (Attribution 4.0 International, as described at https://creativecommons.org/ licenses / by/4.0/). 